\title{
Allograft Inflammatory Factor-1 in Cardiac Ischemia Reperfusion Injury: Release of Molecular Markers in an in Vitro Setting
}

\author{
D. Olga McDaniel ${ }^{1,2 *}$, Xinchun Zhou ${ }^{2}$, Debbie A. Rigney ${ }^{1}$, Larry S. McDaniel ${ }^{3}$, Giorgio Aru', \\ Curtis Tribble $^{1}$, Lawrence Creswell ${ }^{1}$, Walter $\mathrm{H}$. Merrill ${ }^{1}$ \\ ${ }^{1}$ Department of Surgery, University of Mississippi Medical Center, Jackson, USA \\ ${ }^{2}$ Department of Pathology, University of Mississippi Medical Center, Jackson, USA \\ ${ }^{3}$ Department of Microbiology, University of Mississippi Medical Center, Jackson, USA \\ Email: "omcdaniel@umc.edu
}

Received October 25, 2012; revised November 27, 2012; accepted December 9, 2012

\begin{abstract}
Initial ischemia/reperfusion injury (IRI) may have an impact on recipient immune responses after transplantation. Allograft inflammatory factor-1 (AIF-1) has been implicated in the regulation of inflammation associated with organ rejection. We hypothesized that it is either passively released from injured tissues during organ procurement, or actively secreted by allograft infiltrating cells contributing to allograft dysfunction. We investigated the impact of IRI in an in vitro study of human heart tissue during the process of transplantation. The mRNA expression levels for both isoforms of the AIF-1, I2 and I3 were significantly increased after 30 minutes reperfusion (AIF-1 I2: p < 0.01 vs. AIF-1 I3: $p<$ 0.005). Expression levels for IL-18 and the TLRs were increased after 30 minutes of reperfusion. Only IL-18 and TLR-2 were statistically significant (IL-18: $\mathrm{p}<0.0001$ vs. TLR-2: $\mathrm{p}<0.01$ ). The mRNA expression levels for AIF-1 I2 and IL-18 were decreased from the original levels of ischemia after 60 and 90 minutes reperfusion. The TLR-2 and -4 were presented with minimal levels of reduction after 60 minutes. However, mRNA expression levels for all were decreased to the original levels of ischemia after 90 minutes, except for AIF-1 I3, but the difference was not statistically significant. AIF-1 and IL-18 were specifically detected in myocytes and interstitial tissues by immunohistochemistry (IHC) stain after IRI. TLR-4 was non-specific, and TLR2 was minimally expressed. The study discusses the evidence supporting that the AIF-1 may have therapeutic potential for strategies in the control of innate immune responses early on, after transplantation.
\end{abstract}

Keywords: Allograft Inflammatory Factor-1; Cardiac Myocytes; Innate Immunity; Ischemia/Reperfusion; Rejection; Toll-Like Receptors

\section{Introduction}

Advances in surgical techniques, tissue preservation, histocompatibility matching and effective immunosuppression regimens have made cardiac transplantation a successful treatment of choice for individuals with end stage cardiac disease (ESCD). However, antigen independent inflammatory mechanisms have recently been implicated in both immediate and long-term allograft dysfunction and rejection [1]. Immunologically, functional activity of a transplanted organ ultimately depends on the balance between tissue damage caused by ischemia reperfusion (IR), and the recipient immune responses. The early mechanism that triggers rejection episodes (RE) may involve activation of the recipient innate immune response to IR injury (IRI) that occurs during organ pro-

\footnotetext{
"Corresponding author.
}

curement. An innate immune response would cause the release of proinflammatory cytokines and may initiate an adaptive alloimmune response, contributing to allograft dysfunction and early RE. It has been well documented that ischemia (when arterial blood flow is interrupted) creates a structural and metabolic/cellular destruction [2, $3]$, causing cardiac myocyte contraction and tissue damage. Additionally, restoration of blood flow during the reperfusion of the donor organ, although needed it further causes tissue injury. The cytokine associated pathways in IRI may play a significant role in initiating the damage recovery which is critical in long term allograft function. The molecular mechanism underlying IRI is not fully understood.

Studies have shown that inflammation plays an influential role in myocardial IRI and the reperfusion after ischemia causes myocardial injury and cell death $[3,4]$. 
In addition, it has been shown in animal models that production of reactive oxygen species (ROS) during reperfusion affects the severity of injury, causing apoptotic cell death in ischemia regions $[5,6]$. However, the use of antioxidants in clinical studies during reperfusion has not been effective in preventing the IRI [7], suggesting that other mechanisms could be involved in the pathogenesis of IRI. Given the importance of innate immune responses in the recognition of pathogen or damaged tissue associated molecular patterns known as PAMPs or DAMPs respectively, we postulated that the molecules released from cardiomyocytes during IRI, including allograft inflammatory factor-1 (AIF-1) may signal through the toll-like receptors (TLRs), priming the innate effector cells. In cardiac transplantation settings, the signaling of the TLRs, either on donor myocytes or recipient monocytes/dendritic cells entering the graft, ultimately could cause the release of proinflammatory cytokines, leading to allograft dysfunction and RE. An adapted hypothetical model is illustrated in Figure 1 [8].

Allograft inflammatory factor-1 (AIF-1), an evolutionary conserved structural protein has been implicated in the regulation of inflammation and was recently shown to be associated with cellular rejection (CR) after transplantation. AIF-1 is a $17-\mathrm{KDa}$ cytoplasmic, $\mathrm{Ca}^{++}$ binding protein, which is inducible by several cytokines primarily by IFN- $\gamma$, IL- $1 \beta$, IL-18 as well as T cell conditioned media $[9,10]$. This suggests a fundamental role of AIF-1 in the processing of the inflammatory responses. AIF-1 was originally identified and cloned from rat cardiac allografts with chronic rejection [11]. Subsequently, it was identified in human and since then it has been shown that AIF-1 is involved in many pathological processes but is expressed primarily in dendritic cells and macrophages [12]. Nonetheless, it has been found in many other types of cells including activated $\mathrm{T}$ cells $[10,13]$, blood vessel smooth muscle cells after a balloon injury [14], a subset of brain microglial cells [15], vascular endothelial cells [16], devascularized skeletal muscle tissues [17], Langerhan's cells [18], umbilical cord blood stem cells [19], kidney podocytes [20], and spermatides [21]. However, a timely release has not yet been reported for AIF-1 in human cardiac allograft tissue specimens. In this study we investigated release of AIF-1 and the associated markers of the innate immunity, in an in vitro setting using waste tissues excised from cardiac atrium immediately before transplantation. In addition, we tested the presence of these markers in cardiac biopsy samples and in peripheral blood mononuclear cells (MNCs) in association with severity of rejection episodes.

\section{Materials and Methods}

\subsection{Patients and Specimen Collection}

Informed consent was obtained as part of the protocol approved by the University of Mississippi Medical Center (UMMC) Review Board for obtaining blood samples and biopsy specimen from each patient. All patients had undergone cardiac transplantation at UMMC. Blood and biopsy samples were obtained at the same time. Blood samples and biopsy specimen from the same patientswho had undergone cardiac allograft transplantation were

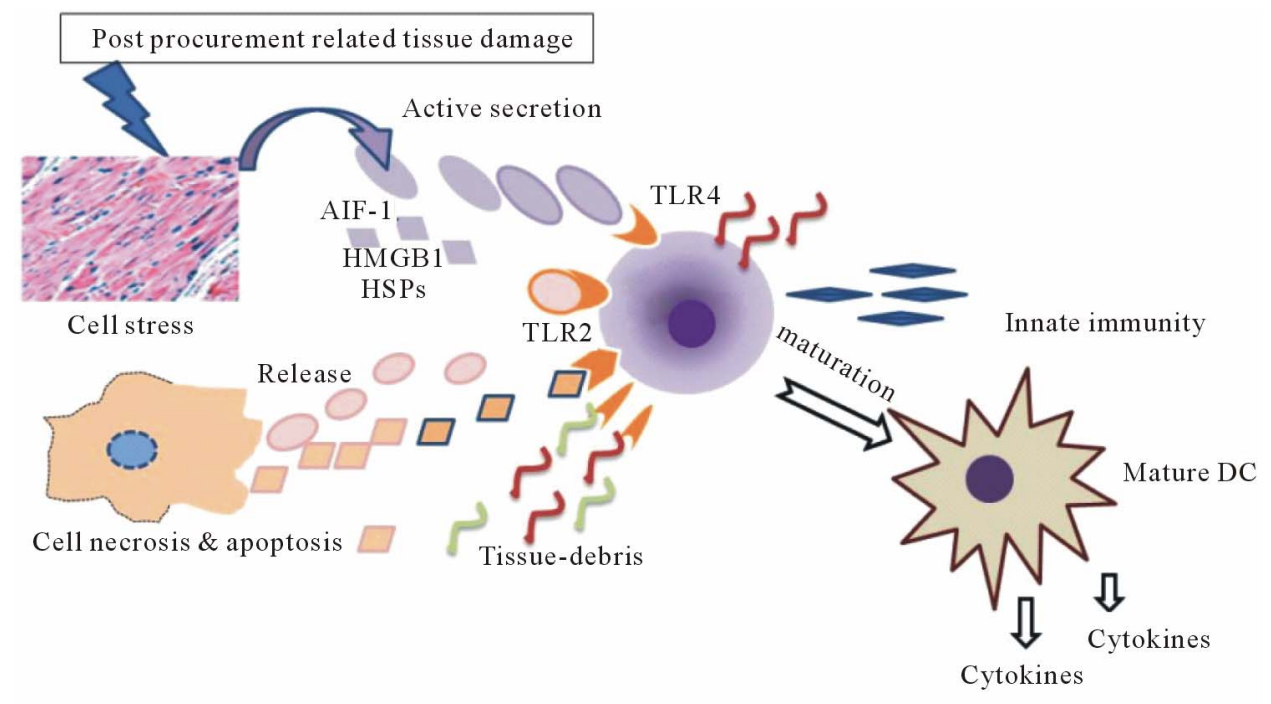

Rejection $\longleftarrow$ Organ dysfunction $\longleftarrow$ Alloreactivity $\longleftarrow$ Adaptive

Figure 1. An oversimplified schematic illustration of ischemia reperfusion injury (IRI) is described. A series of signaling event occurs in which the AIF-1 released from the donor organ acts as a ligand stimulating the innate immune effector cells through the Toll-like receptors (TLRs), causing the release of proinflammatory cytokines, potentiating the development of adaptive alloimmunity leading to organ dysfunction and rejection episodes. 
selected from our sample depository for the study. Patients were classified into testing groups based on endo myocardial biopsy and histopathological assessments for rejection scores: Grade $0(0 \mathrm{R})$, Grade $1 \mathrm{~A}(\mathrm{R})$, Grade 2, Grade 3A (2R), and 3A/3B (3R) according to an established criterion by the International Society for Heart and Lung Transplantation (ISHLT) [22]. A total of 30 patients, 10 in a group with Grade 0 rejection; 10 in a group with $1 \mathrm{~A}$ rejection and 10 in a group with $3 \mathrm{~A}$ rejection were included in this study. Allograft tissues from atrium that was considered trimmings or waste were collected immediately after removal of the tissue in the operating room for in vitro ischemia reperfusion studies.

\subsection{In Vitro Ischemia/Reperfusion (IR)}

The trimmings from allograft atrium were utilized to set up the in vitro the IR procedure. Specimens before treatment were considered ischemic time point. Tissues were cut in $1 \times 2$ square $\mathrm{mm}$, some stored either in formaldehyde for paraffin block preparation for IHC stain, or in $-80^{\circ} \mathrm{C}$ freezer to be used for RNA preparation. The remaining squares were subjected to reperfusion experiments. Tissues were washed with phosphate buffered saline (PBS) and incubated for 30 - 90 minutes time intervals in a $5 \%$ glucose-ringers saline medium in an aerobic chamber with $5 \% \mathrm{CO}_{2}$ at $37^{\circ} \mathrm{C}$ incubator. At the end of the treatment, tissues were collected and placed either in formaldehyde or stored at $-80^{\circ} \mathrm{C}$.

\subsection{RNA Isolation and Reverse-Transcription Polymerase Chain Reaction (RT-PCR)}

To determine expression levels for AIF-1, TLR-2, TLR-4 and IL-18, RNA was isolated from peripheral blood mononuclear cells (PBMCs), monocytes and cardiac tissue specimens. Briefly, monocytes were isolated from patient's PBMCs after a Ficoll-paque density gradient and positive selection for CD14-positive cells with Dynal beads. Cells were immediately homogenized in TRIzol and were stored at $-80^{\circ} \mathrm{C}$ for subsequent RNA extraction. RNA was extracted from the cells using a kit from Invitrogen (Invitrogen Corp. Carlsbad, CA, USA), according to the manufacturer guidelines. RNA concentration and purity were determined using NanoDrop 2000C (Thermo-Fisher, GA). Also, frozen tissue specimens were homogenized in $200 \mu \mathrm{l}$ cell lysate buffer for RNA extraction using a kit from Invitrogen (Invitrogen Corp. Carlsbad, CA, USA), according to the manufacturer's guidelines. Afterwards RNA was transcribed by the ImProm-II Reverse Transcription method (Promega Incorporation, Madison, WI), followed by a routine RT-PCR protocol using appropriate amplification primers given in Table 1. AIF-1 has three isoforms which are generated due to the gene splicing. Originally we tested all three isoforms to determine the level of association with rejection episodes. The orientation of the three isoforms is given in Figure 2(A). The isoforms 1 and 2 are identical at $\mathrm{N}$-terminal, thus, we chose the same 5' forward

Table 1. Primer sequences (5'-3') for amplification of mRNA expression levels.

\begin{tabular}{|c|c|c|}
\hline Genes & Forward Primer/Reverse Primer & Size Base-Pair \\
\hline \multicolumn{3}{|l|}{ AIF-1 } \\
\hline \multirow[t]{2}{*}{ Isoform1 } & 5 '-atggagtttgaccttaatgg-3' & 278 \\
\hline & 5'-gcaactcagagatagctttg-3' & \\
\hline \multirow[t]{2}{*}{ Isoform 2} & 5'-atggagtttgaccttaatgg-3' & 397 \\
\hline & 5'-tcacatttttaggatggcagatc-3' & \\
\hline \multirow[t]{2}{*}{ Isoform 3} & $5^{\prime}$ - atggagtttgaccttaatgg- $3^{\prime}$ & 438 \\
\hline & 5'- gcaactcagagatagctttg-3' & \\
\hline \multirow[t]{2}{*}{ IL-18 } & 5'-atggctgctgaaccagtag-3' & 550 \\
\hline & 5'-ctagtcttcgttttgaaca-3' & \\
\hline \multirow[t]{2}{*}{ TLR-2 } & 5'-gccaaagtcttgattgattgg-3' & 387 \\
\hline & $5^{\prime}$-ttgaagttctccagctcctg-3' & \\
\hline \multirow[t]{2}{*}{ TLR-4 } & $5^{\prime}$-tgctgccaacatcatcca-3' & 303 \\
\hline & $5^{\prime}$-atggatgatgatatcgccgcg-3' & \\
\hline \multirow[t]{2}{*}{ beta-Actin } & $5^{\prime}$-atggatgatgatatcgccgcg-3' & 1078 \\
\hline & 5'-ctagaagcatttgcggtggacgat-3' & \\
\hline
\end{tabular}

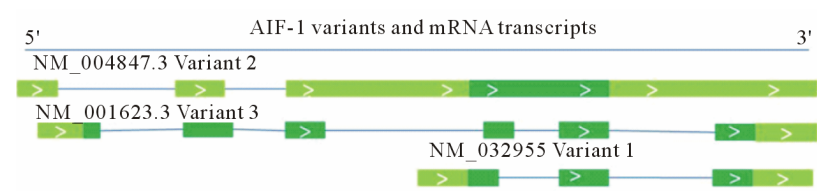

(A)

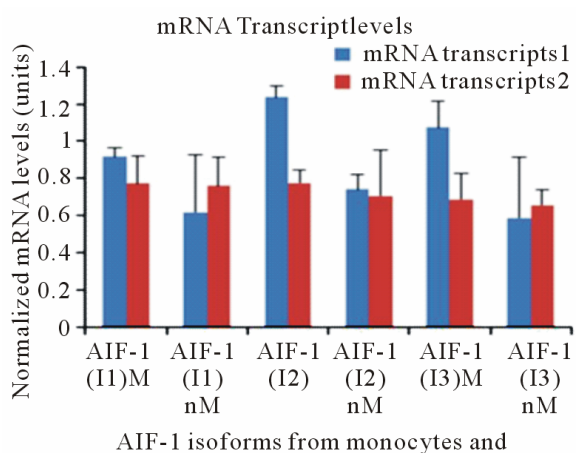
monocyte depleted PBMCs

(B)

Figure 2. A diagram of AIF-1 variants (Isoforms) and mRNA transcripts are illustrated. (A) The three isoforms are generated by gene splicing and have a partial sequence homology. The isoforms 1 and 2 are identical at $\mathrm{N}$-terminal, and the isoform 1 and 3 have an identical C-terminal; (B) mRNA transcript levels calculated after they were normalized to $\beta$-Actin expression levels. 
primers, and the isoforms 1 and 3 have an identical Cterminal, thus, we chose the same 3 'reverse primers for both. The amplified PCR products for expression levels of mRNA transcripts were visualized by $2.0 \%$ agarose gel. The intensity of the amplified banding pattern for each gene was quantified using a GDS-8000 (UVP) Image Analysis System and the level was normalized to the $\beta$-Actin gene expression levels. The normalized values (Unit) were presented as mean $\pm \mathrm{SEM}$.

\subsection{Immunohistochemistry (IHC)}

The formalin fixed paraffin embedded (FFPE) tissue sections were utilized in preparation of tissue arrays. A pre-made paraffin recipient block was used to prepare the array. The area of interest on Hematoxylin and Eosin $(\mathrm{H} \& \mathrm{E})$ stain were matched to the donor block and the tissue was cored and deposited into a 48 wells recipient block. The recipient blocks were treated at $40^{\circ} \mathrm{C}$ and then chilled at $4^{\circ} \mathrm{C}$ to generate a smooth paraffin surface appropriate for sectioning. Serial $4-8 \mathrm{~mm}$ thick sections were prepared for IHC stain. The slides were then deparaffinized in Xylene, and rehydrated through a graded series of ethanol followed by treatment with 3\% hydrogen peroxide in phosphate buffered saline (PBS) to block the endogenous peroxidase activity. After antigen retrieval with Citra plus and blocking with normal serum provided in the $\mathrm{ABC}$ kit, sections were incubated with primary antibodies against AIF-1 [23] or IL-18, TLR-2, or TLR-4 as described previously [24].

\subsection{Data Analyses}

The ISHLT grades for each biopsy specimen were analyzed for the presence or absence of AIF-1, IL-18, TLR-2 and TLR-4. Both interstitial infiltrating MNCs and CMCs were evaluated for IHC staining. The RNA transcript results were expressed as mean \pm SEM. Group differences among mRNA transcript levels between the rejection grades were determined by one-way analysis of variance. The level of significance was set at $p<0.05$ for comparison between the groups.

\section{Results}

A total of 70 paraffin blocks of cardiac tissues or biopsy specimens from the patients who had undergone cardiac allograft transplantation were tested for AIF-1, IL-18, TLR-2 and TLR-4 gene expression. In addition, peripheral blood PBMCs and MNCs corresponding with the time when allograft biopsy was taken were tested for expression levels of mRNA transcripts. The test samples were normalized to $\beta$-Actin expression levels.

\subsection{MRNA Gene Expression Levels for Each AIF-1 Isoforms}

To determine which of the 3 AIF-1 isoforms were asso- ciated with graft rejection the mRNA expression levels of the three isoforms, 1 (I1), 2 (I2) and 3 (I3) were tested in the PBMCs from recipients who had experienced rejection episodes. The PBMCs from the same recipients at the time when they were rejection free were used as a control for comparison analysis. As shown in Figure 2(B), AIF-1 isoform 2 was significantly increased in association with the ISHLT grade $3 \mathrm{~A}$ rejection as compared with grade 0-1A. Isoform 3 was also increased in association with RE but statistically were less significant (I2: $p<0.005$ vs. I3: $p<0.06$ ). Subsequently, only AIF-1 I2 and I3 mRNA transcripts were tested in tissue specimens that had undergone ischemia reperfusion experiments.

\subsection{Evaluation of the mRNA Transcript at Different Time Intervals of Reperfusion}

As shown in Figure 3(A), mRNA expression levels for

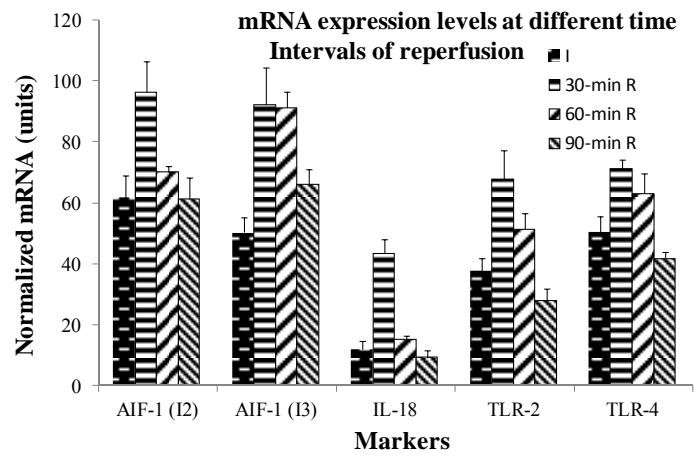

(A)

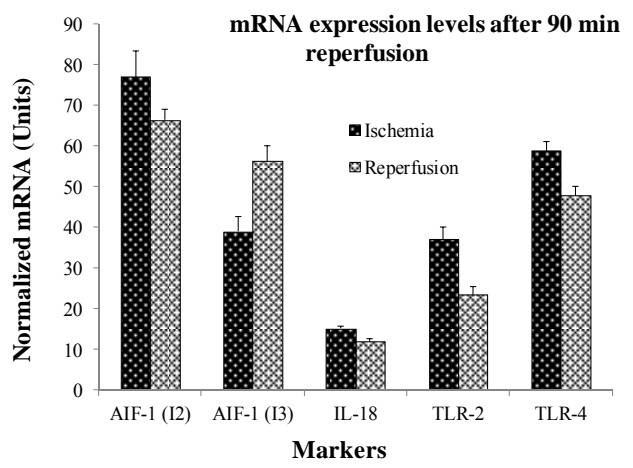

(B)

Figure 3. Variations in mRNA expression levels in tissue specimen are shown after ischemia/reperfusion. (A) mRNA transcripts at different time intervals of reperfusion. AIF-1 isoform 1 and 2 were significantly increased after 30 minutes of reperfusion (I2: $p<0.01$ vs. I3: $p<0.005$ ). Only the IL-18 and TLR-2 expression levels were statistically significant (IL-18: p < 0.0001 vs. TLR-2: p < 0.01); (B) mRNA transcripts were evaluated in an additional samples for the effects of 90 minutes reperfusion. I3 was increased 1.4-fold after reperfusion $(p<0.05)$, whereas the others were decreased. A $34.3 \%$ and $20.6 \%$ reduction was observed for TLR-2 and TLR-4 respectively after reperfusion. 
both isoforms of the AIF-1, I2 and I3 were significantly increased after 30 minutes of reperfusion (AIF-1 I2: $p<$ 0.01 vs. AIF-1 I3: $p<0.005$ ). Since AIF-1 is inducible with IL-18 and it has been shown to be involved in organ IRI, we then tested the levels of IL-18 mRNA expressions in addition to the AIF-1, TLR2 and TLR4. The mRNA expression levels for IL-18 and the TLRs were increased after 30 minutes of reperfusion. Only IL-18 and the TLR-2 were statistically significant (IL-18: $\mathrm{p}<$ 0.0001 vs. TLR-2: $\mathrm{p}<0.01$ ). The mRNA expression levels for AIF-1 I2 and IL-18 were decreased from the original levels of ischemia after 60 and 90 minutes reperfusion. The TLR-2 and -4 were presented with minimal levels of reduction after 60 minutes. However, mRNA expression levels for all were decreased to the original levels of ischemia after 90 minutes, except for AIF-1 I3, but the difference was not statistically significant.

\subsection{MRNA Transcript Evaluation after 90 Minutes Reperfusion}

We examined mRNA expression levels in an additional 10 cardiac allograft samples for the impact of ischemia and reperfusion after 90 minutes. The mRNA expression levels for AIF-1 I3 was increased 1.4-fold after 90 minutes reperfusion $(p<0.05)$. For the TLR-2 and -4 , the mRNA expression levels were significantly decreased after 90 minutes. There was a $34.3 \%(p<0.05)$, and a $20.6 \%$ reduction in expression levels of TLR-2 and TLR-4 respectively after reperfusion. The levels of mRNA expression for AIF-1, I2 and IL-18 were decreased but not statistically significant (Figure 3(B)).

\subsection{Cellular Distribution of AIF-1, IL-18, and TLRs}

A strong immunoreactivity observed for AIF-1 and IL-18 markers in both ischemic tissues and in tissues after reperfusion. AIF-1 and IL-18 stain were present in both myocytes nucleus and in interstitial cells. The rates of immunoreactivity for AIF-1 and IL-18 were associated with the time length of the reperfusion (Figure 4). There was a minimal immunoreactivity with anti-TLR-2 detected in both ischemic and after 90 minutes reperfusion tissue specimens. On the other hand, a strong TLR-4 immunoreactivity was observed in both myocyte nucleus and myocyte fibers as well as interstitial segments (Figure 5). The rates of immunoreactivity were associated with the length of reperfusion.

\section{Discussion}

It has been documented that during ischemia reperfusion damage, rays of tissue debris and cellular elements are released which may have impact on the recipient immune response to allograft causing the dysfunction of the organ

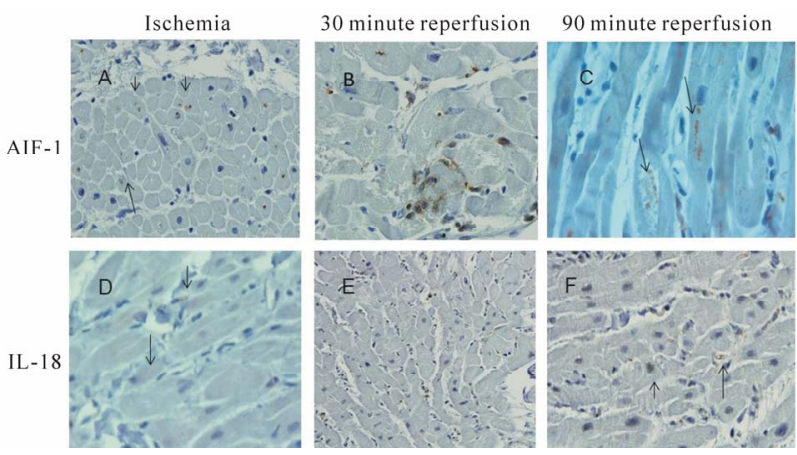

Figure 4. IHC stains of cardiac specimens tested with AIF-1 and IL-18 antibody. (A) Ischemic tissue tested with AIF-1 antibody; (B) After 30 minute reperfusion; (C) After 90 minutes reperfusion; (D) Ischemic tissue tested with IL-18 antibody; (E) After 30 minute reperfusion; (F) After 90 minute reperfusion. Brown stains present positive reactivity.
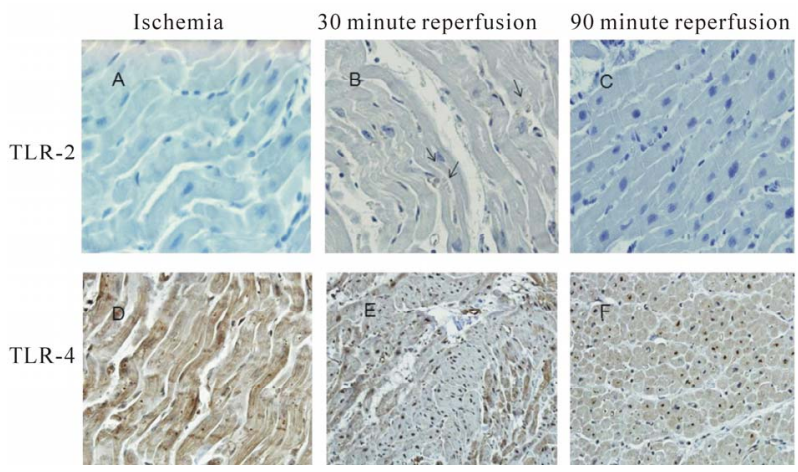

Figure 5. IHC stains of cardiac specimen tested TLR-2 and TLR-4 antibody. (A) Ischemic tissue tested with TLR-2 antibody; (B) After 30 minute reperfusion; (C) After 90 minutes reperfusion; (D) Ischemic tissue tested with TLR-4 antibody; (E) After 30 minute reperfusion; (F) after 90 minute reperfusion. Brown stains present positive reactivity.

and may lead to allograft rejection $[1,25]$. In addition, molecular markers associated with innate immunity have been implicated in the regulation of inflammatory response during IR [26,27]. In this study we have generated in vitro IR settings to observe presence of molecular markers associated with the initial inflammatory response that may trigger the innate immune response after cardiac transplantation. The practical value of this study was the findings that the AIF-1, IL-18 and the TLRs that were previously shown in the peripheral blood and biopsy specimen from allograft recipients with $3 \mathrm{~A}$ rejection were also present in the cardiac tissues during the IR. This suggests that these markers may act as the initial sensors for innate immune response and may provide key elements to the genesis of post transplantation alloreactivity to the allograft. Furthermore, it suggests that a possible blockage of these markers may have a therapeutic significance during organ procurement by providing myocardial protection. 
The data in this study have shown for the first time a direct evidence for the presence of AIF-1, IL-18 and TLRs in human donor cardiac tissue immediately after transplantation. In addition, we have demonstrated variations in the level of expression at different time intervals in an in vitro reperfusion setting. Considering the fact that AIF-1, IL-18, TLR-2 and -4 have shown to be upregulated during cardiac allograft rejection [24,28,29], and there is a suggestive evidence that AIF-1 may signal through the TLRs, it was important to seek such evidence during the IR. In transplantation settings it was suggested that the TLRs are either on donor myocytes, or on recipient mononuclear cells recruited to the heart after transplantation. We have shown a strong positive immunoreactivity with anti-TLR-4, in cardiac tissue after 30 minutes reperfusion and it remained positive through the 90 minutes of the experiment's end point. We suggest that increased TLR-4 in cardiac tissue during the IR triggers the inflammation through the TLR-signaling pathway in association with the molecules such as AIF-1 released from cardiac tissue due to IRI. This event causes production of proinflammatory cytokines which could have impact on allograft dysfunction, leading to allograft rejection.

Previous studies have shown that AIF-1 plays a pivotal role in survival and in the inflammatory pathway of macrophages [30]. The level of AIF-1 expression in rat macrophages has been shown to be augmented by IFN-gamma and IL-1 $\beta$ [9]. It was also shown that blocking AIF-1 expression reduced macrophage migration and proliferation [31]. Furthermore, in a rat model of IRI, the levels of AIF-1 expression were paralleled with the expression levels of IFN-gamma after reperfusion, suggesting the molecular involvement of the AIF-1 in proinflammatory response of the macrophages. However, in the present study, we were unable to demonstrate expression of IFN-gamma in human cardiac tissue after in vitro IR, neither with RT-PCR nor IHC stain. The immunologic function of AIF-1 is still not clearly known, although a variety of pathologic processes including allograft rejection and vasculaopathy have demonstrated the presence and the involvement of AIF-1 [23,24,28,32]. Autieri et al., have shown the impact of cell cycle deregulation in over expression of AIF-1, causing proliferation of vascular smooth muscle cells [16]. The AIF-1 expression level was shown to be upregulated in cardiac allograft after cold and warm IR and the expression was reduced with a low dose of FK506 pretreatment [33]. This finding suggests that the FK506 effect most likely was not on the recipient macrophages infiltrating the allograft, but was on donor organ tissue. In such case the FK506 probably blocked the receptors on cardiac myocytes, preventing the underlying mechanisms involved in pathogenesis of IRI. Recent studies reported that shortterm treatment of skin with Tacrolimus caused a reduc- tion in both TLR-1 positive keratinocytes and dermal infiltrating cells, but have increased the dermal TLR-2 positive cells in atopic dermatitis (AD), affecting the inflammation associated with $\mathrm{AD}$ [34]. Although the role of TLRs was not discussed in the previous report [33], there is suggestive evidence that FK506 may inhibit the AIF-1 expression through the TLRs. In our study, the mRNA expression levels of TLR-2 and TLR-4 were increased after 30 minutes reperfusion, and the TLR4 expression level remained high after 60 minutes. However, the TLR-2 positive myocytes were minimal, but the TLR-4 positive myocytes were considerably increased (Figure 5), which may support the role that they play in IRI. Our in vitro study has limitation which would not provide any evidence of functional interaction between these molecules. However, it supports the presence of inflammatory markers associated with early molecular events that activates the innate system after IRI.

TLRs play a major role in the activation of innate immune responses, through the TLR-signaling pathway in association with PAMPs or molecules that are released from damaged tissues, or cell death due to injury, disease and age. Activation of the TLRs has been associated with IRI, delayed graft function, and allograft rejection [35, 36]. Furthermore, inhibition of TLR-2 has been shown to provide a significant protection from IRI in a mouse model of kidney transplantation [37], and myocardial infarction [38]. Therefore we reasoned that because of the involvement of these molecules in innate immune responses, it would be worthy to investigate the expression levels of these markers in an in vitro experiment with the notion that identification of AIF-1 and IL-18 in association with the TLRs during IR would support the hypothesis that these markers together contribute to the initial process of inflammation. Additional in vitro and in vivo studies for verification of the role of AIF-1 as an endogenous ligand as well as the TLRs will be needed to confirm the association between these molecules during the initial innate immune responses in transplantation settings. These studies are in progress. Primary cell lines generated from human cardiac tissue has been utilized for production of AIF-1, and in association with cell lines transfected with TLRs, the investigation is seeking to demonstrate the interactions between these molecules and the impact on the production of proinflammatory cytokines and chemokines. It is our goal that antagonizing at least one or any of these molecules before reperfusion could protect cardiac function, and may prevent early allograft dysfunction and RE.

\section{Conclusion}

The in vitro model of ischemia/reperfusion showed that human donor cardiac tissue produces an increased level of AIF-1, IL-18 and TLR-4 during IR. This suggests their 
participation in innate immune recognition of cardiac allograft. Further, they may have therapeutic characteristics for strategies in the control of innate immune responses in allograft transplantation.

\section{Acknowledgements}

The authors thank the patients who participated in this study and the staff of the Cardiac Catheterization Laboratory at the University Mississippi Medical Center (UMMC) for their assistance in obtaining blood samples and endomyocardial biopsy specimens. This work was supported by Transplantation Immunogenetics Laboratory in the Department of Surgery at UMMC.

\section{REFERENCES}

[1] W. G. Land, "Innate Immunity-Mediated Allograft Rejection and Strategies to Prevent It," Transplantation Proceedings, Vol. 39, No. 3, 2007, pp. 667-672. doi:10.1016/j.transproceed.2007.01.052

[2] J. Gurevitch, I. Frolkis and Y. Yuhas, "Tumor NecrosisAlpha Is Released from the Isolated Heart Undergoing Ischemia and Reperfusion," The American College of Cardiology, Vol. 28, No. 1, 1966, pp. 247-252. doi:10.1016/0735-1097(96)00105-2

[3] D. M. Yellon and D. J. Hausenloy, "Mechanisms of Disease: Myocardial Reperfusion Injury," The New England Journal of Medicine, Vol. 357, No. 9, 2007, pp. 11211135. doi:10.1056/NEJMra071667

[4] W. G. Land, "The Role of Postischemic Reperfusion Injury and Other Nonantigen-Dependent Inflammatory Pathways in Transplantation," Transplantation, Vol. 79, No. 5, 2005, pp. 505-514. doi:10.1097/01.TP.0000153160.82975.86

[5] J. M. Downey, "Free Radicals and Their Involvement during Long-Term Myocardial Ischemia and Reperfusion," Annual Review Physiology, Vol. 52, 1990, pp. 487504.

[6] E. Noiri, A. Nakao, K. Uchida, et al., "Oxidative and Nitrosative Stress in Acute Renal Ischemia," American Journal of Physiological Renal Physiology, Vol. 281, No. 5, 2001, pp. F948-F957.

[7] J. T. Flaherty, B. Pitt, J. W. Gruber, et al., "Recombinant Human Superoxide Dismutase (h-SOD) Fails to Improve Recovery of Ventricular Function in Patients Undergoing Coronary Angioplasty for Acute Myocardial Infarction," Circulation, Vol. 89, No. 5, 1994, pp. 1982-1991. doi:10.1161/01.CIR.89.5.1982

[8] W. G. Land, "Emerging Role of Innate Immunity in Organ Transplantation Part II: Potential of Damage-Associated Molecular Patterns to Generate Immunostimulatory Dendritic Cells," Transplantation Reviews, Vol. 26, No. 2, 2012, pp. 73-87. doi:10.1016/j.trre.2011.02.003

[9] G. Liu, H. Ma, L. Jiang and Y. Zhao, "Allograft Inflammatory Factor-1 and Its Immune Regulation," Autoimmunity, Vol. 40, No. 2, 2007, pp. 95-102. doi:10.1080/08916930601083946
[10] F. Del Galdo and S. A. Jimenez, "T Cell Expressing Allograft Inflammatory Factor-1 Display Increases Chemotaxis and Induce a Pro-Fibrotic Phenotype in Normal Fibroblasts in Vitro," Arthritis Rheumatism, Vol. 56, No. 10, 2007, pp. 3478-3488. doi:10.1002/art.22877

[11] U. Utans, R. J. Arceci, Y. Yamashita and M. E. Russell, "Cloning and Characterization of Allograft Inflammatory Factor-1: A Novel Macrophage Factor Identified in Rat Cardiac Allografts with Chronic Rejection," Journal of Clinical Investigation, Vol. 95, No. 5, 1995, pp. 29542962. doi:10.1172/JCI118003

[12] U. Utans, W. C. Quist, B. M. McManus, J. E. Wilson and R. J. Arceci, "Allograft Inflammatory Factor-1. A Cytokine Responsive Macrophage Molecule Expressed in Transplanted Human Hearts," Transplantation, Vol. 61, No. 9, 1996, pp. 1387-1392. doi:10.1097/00007890-199605150-00018

[13] S. E. Kelemen and M. V. Autieri, "Expression of Allograft Inflammatory Factor-1 in T Lymphocytes: A Role in T-Lymphocyte Activation and Proliferative Arteriopathies," American Journal of Pathology, Vol. 167, No. 2, 2005, pp. 619-626. doi:10.1016/S0002-9440(10)63003-9

[14] M. V. Autieri, C. Carbone and A. Mu, "Expression of Allograft Inflammatory Factor-1 Is a Marker of Activation Human Vascular Smooth Muscle Cells and Arterial Injury," Arteriosclerosis Thrombosis Vascular Biology, Vol. 20, No. 7, 2000, pp. 1737-1744. doi:10.1161/01.ATV.20.7.1737

[15] M. H. Deininger, K. Seid, S. Engel, R. Meyermann and H. J. Schluesener, "Allograft Inflammatory Factor-1 Defines a Distinct Subset of Infiltrating Macrophages/microglial cells in Rat and Human Gliomas," Acta Neurophathology, Vol. 100, No. 6, 2000, pp. 673-680. doi:10.1007/s004010000233

[16] Y. Tian, S. Jain, S. E. Kelemen and M. V. Autieri, “AIF-1 Expression Regulates Endothelial Cell Activation, Signal Transduction, and Vasculogenesis," American Journal of Physiology Cell Physiology, Vol. 296, No. 2, 2009, pp. C256-C266. doi:10.1152/ajpcell.00325.2008

[17] R. Kuschel, M. H. Deininger, R. Meyermann, A. Bornemann, Z. Yablonka-Reuveni and H. J. Schluesener, "Allograft Inflammatory Factor-1 Is Expressed by Macrophages in Injured Skeletal Muscle and Abrogates Proliferation and Differentiation of Satellite Cells," Journal of Neurophatology Experimental Neurology, Vol. 59, No. 4, 2000, pp. 323-332.

[18] C. Orsmark, T. Skoog, L. Jeskanen, J. Kere and U. Saarialho-Kere, "Expression of Allograft Inflammatory Factor1 in Inflammatory Skin Disorders," Acta Dermato-Venereologica, Vol. 87, No. 3, 2007, pp. 223-227.

[19] T. C. Lund, L. B. Anderson, V. McCullar, et al., "iTRAQ Is a Useful Method to Screen for Membrane-Bound Proteins Differentially Expressed in Human Natural Killer Cell Types," Journal of Proteome Research, Vol. 6, No. 2, 2007, pp. 644-653. doi:10.1021/pr0603912

[20] Y. Tsubata, M. Sakatsume, A. Ogawa, et al., "Expression of Allograft Inflammatory Factor-1 in Kidneys: A Novel Molecular Component of Podocyte," Kidney International, Vol. 70, No. 11, 2006, pp. 1948-1954. 
[21] C. Köhler, "Allograft Inflammatory Factor-1/Ionized Calcium-Binding Adapter Molecule 1 Is Specifically Expressed by Most Subpopulations of Macrophages and Spermatids in Testis," Cell and Tissue Research, Vol. 330, No. 2, 2007, pp. 291-302. doi:10.1007/s00441-007-0474-7

[22] D. O. Taylor, L. B. Edwards, M. M. Boucek, et al., "Registry of the International Society for Heart and Lung Transplantation: Twenty-Second Official Adult Heart Transplant Report, 2005," Journal of Heart Lung Transplantation, Vol. 24, No. 8, 2005, pp. 945-955. doi:10.1016/i.healun.2005.05.018

[23] X. Zhou, Z. He, J. Henegar, B. Allen and S. Bigler, "Expression of Allograft Inflammatory Factor-1 (AIF-1) in Acute Cellular Rejection of Cardiac Allografts," Cardiovascular Pathology, Vol. 20, No. 5, 2011, pp. e177-e184. doi:10.1016/j.carpath.2010.08.002

[24] A. K. Barker, D. O. McDaniel, X. Zhou, et al., "Combined Analysis of Allograft Inflammatory Factor-1, Interleukin-18, and Toll-Like Receptor Expression and Association with Allograft Rejection and Coronary Vasculopathy," The American Surgeon, Vol. 76, No. 8, 2010, pp. 872-878.

[25] A. M. Sheridan and J. V. Bonventre, "Cell Biology and Molecular Mechanisms of Injury in Ischemic Acute Renal Failure," Current Opinion Nephrology Hypertension, Vol. 9, No. 4, 2000, pp. 427-434. doi:10.1097/00041552-200007000-00015

[26] D. J. Kaczorowski, A. Tsung and T. R. Billiar, "Innate Immune Mechanisms in Ischemia/Reperfusion," Frontiers Bioscience, Vol. 1, No. 6, 2009, pp. 91-98.

[27] M. Takahashi, "Role of the Inflammasome in Myocardial Infarction," Trends in Cardiovascular Medicine, Vol. 21, No. 2, 2011, pp. 37-41. doi:10.1016/j.tcm.2012.02.002

[28] M. V. Autieri, S. Kelemen, B. A. Thomas, E. D. Feller, B. I. Goldman and H. J. Eisen, "Allograft Inflammatory Factor-1 Expression Correlates with Cardiac Rejection and Development of Cardiac Allograft Vasculopathy," Circulation, Vol. 106, No. 17, 2002, pp. 2218-2223. doi:10.1161/01.CIR.0000035652.71915.00

[29] D. O. McDaniel, X. Zhou, C. K. Moore and G. Aru, "Cardiac Allograft Rejection Correlates with Increased Expressions of Toll-like Receptor 2 and 4 and Allograft Inflammatory Factor 1," Transplantation Proceedings, Vol. 42, No. 10, 2010, pp. 5235-4237. doi:10.1016/j.transproceed.2010.09.091

[30] Z. F. Yang, D. W. Ho, C. K. Lau, et al., "Allograft Inflammatory Factor-1 (AIF-1) Is Crucial for the Survival and Pro-Inflammatory Activity of Macrophages," International Immunology, Vol. 17, No. 11, 2005, pp. 13911397. doi: $10.1093 /$ intimm/dxh316

[31] Y. Tian, S. E. Kelemen and M. V. Autieri, "Inhibition of AIF-1 Expression by Constitutive siRNA Expression Reduces Macrophage Migration, Proliferation, and Signal Transduction Initiated by Atherogenic Stimuli," American Journal of Physiology Cell Physiology, Vol. 290, No. 4, 2006, pp. C1083-C1091. doi:10.1152/ajpcell.00381.2005

[32] Y. Nagakawa, S. Nomoto, Y. Kato, et al., "Over-Expression of AIF-1 in Liver Allograft and Peripheral Blood Correlates with Acute Rejection after Transplantation in Rats," American Journal of Transplantation, Vol. 4, No. 12, 2004, pp. 1949-1957. doi:10.1111/j.1600-6143.2004.00621.x

[33] W. Jiang, L. Kong, X. Wu and X. Wang, "Allograft Inflammatory Factor-1 Is Up-Regulated in Warm and Cold Ischemia-Reperfusion Injury in Rat Liver and May Be Inhibited by FK506," Journal of Surgical Research, Vol. 165, No. 1, 2011, pp. 158-164. doi:10.1016/j.jss.2009.05.038

[34] E. Antiga, W. Volpi, D. Torchia, P. Fabbri and M. Caproni, "Effects of Tacrolimus Ointment on Toll-Like Receptors in Atopic Dermatitis," Clinical Experimental Dermatology, Vol. 36, No. 3, 2011, pp. 235-241. doi:10.1111/j.1365-2230.2010.03948.x

[35] A. Gluba, M. Banach, S. Hannam, D. P. Mikhailidis, A. Sakowicz and J. Rysz, "The Role of Toll-Like Receptor in Renal Diseases," Nature Review Nephrology, Vol. 6, No. 4, 2010, pp. 224-235. doi:10.1038/nrneph.2010.16

[36] D. R. Goldstein, "Toll Like Receptors and Acute Allograft Rejection," Transplant Immunology, Vol. 17, No. 1, 2006, pp. 11-15. doi:10.1016/j.trim.2006.09.012

[37] C. A. Farrar, B. Keogh, W. McCormack, et al., "Inhibition of TLR2 Promotes Graft Function in a Murine Model of Renal Transplant Ischemia-Reperfusion Injury," Federation American Society Experimental Biology Journal, Vol. 26, No. 2, 2012, pp. 799-807.

[38] F. Arslan, M. B. Smeets, A. A. O’Neill, et al., "Myocardial Ischemia/Reperfusion Injury Is Mediated by Leukocytic Toll-like Receptor- 2 and Reduced by Systemic Administration of a Novel Anti-Toll-Like Receptor-2 Antibody," Circulation, Vol. 121, No. 1, 2010, pp. 80-90. doi:10.1161/CIRCULATIONAHA.109.880187

[39] S. Frantz, L. Kobzik, K. Young-Dae, et al., "Toll4 (TLR4) Expression in Cardiac Myocytes in Normal and Failing Myocardium," Journal of Clinical Investigation, Vol. 104, No. 3, 1999, pp. 271-280. doi:10.1172/JCI6709 